\title{
Quantum Recurrences in Periodically Driven Systems
}

\author{
Farhan Saif \\ Department of Electronics, Quaid-i-Azam University, Islamabad, Pakistan.
}

(October 11, 2018)

\begin{abstract}
We investigate the quantum recurrence phenomena in periodically driven systems. We calculate the classical period and the quantum recurrence time and develop their interdependence. We further predict the behavior of the recurrence phenomena for the power law potentials.

PACS numbers: 03.65.Sq, 05.45.-a, 05.45.Mt, 47.52.+j
\end{abstract}

\section{INTRODUCTION}

The phenomena of recurrence or revival is a beautiful combination of classical mechanics, wave mechanics, and quantum laws. A wave packet evolves over a short period of time in a potential, initially following classical mechanics. It spreads while moving along its classical trajectory, however rebuilds itself after a classical period. It follows wave mechanics in its long time evolution and gradually observes a collapse. However, the discreteness of quantum mechanics leads to the restoration and restructuring of the wave packet.

In one degree of freedom systems the phenomena of quantum revivals are well studied both theoretically and experimentally. The quantum revivals were first studied in cavity quantum electrodynamics [1-3]. Recently, the existence of revivals has been investigated in atomic [4-11] and molecular [12-15] wave packet evolution.

The periodically driven quantum systems [16-18], and two-degree-of-freedom systems such as stadium billiard [19] indicate the presence of quantum revivals in higher dimensional systems. Latter, it is proved that the recurrence or revival phenomenon is a generic property of the one degree of freedom periodically driven quantum systems [20]. In the present contribution we calculate the classical period and quantum revival time for the driven systems. Moreover, we calculate their interdependence for different dynamical regimes. Latter we explain these interdependences for the power law potentials.

The layout of the paper is as follows: In Sec. II, we write general Hamiltonian for the periodically driven time dependent systems. In Sec. III, we calculate the quasi-energy eigen functions and quasi-energies for these systems. In Sec. IV we calculate the classical period and quantum recurrence time and write their interdependence in Sec. V. We dedicate section VI for a discussion of this interdependence in power law potentials.

\section{PERIODICALLY DRIVEN SYSTEMS}

In its evolution in time a material wave packet displays quantum revivals in a one dimensional system driven by an external periodic force [20]. Subject to the strength of the external modulation, the classical dynamics may support stable and unstable motion. The Hamiltonian of the system in the dimensionless form is expressed as,

$$
H=H_{0}+\lambda V(z) \sin t
$$

Here, $H_{0}=p^{2} / 2 m+V_{1}$, is the Hamiltonian of the undriven system, and $V_{1}$ is the potential of the undriven system. The eigen states and the eigen values of the time independent system are, $|n\rangle$, and, $E_{n}$, respectively. Moreover, $\lambda$ expresses the dimensionless modulation strength, and $V(z)$ defines the coupling between the one dimensional potential and the external modulation.

The measurement of the time of the revival in the classical domain and in the quantum domain requires the solution of the periodically driven quantum system. In the time dependent system the energy is no more a constant of motion. Therefore, the periodically driven system is made to solve in the region of resonances by using the secular perturbation theory [21]. In this approach, the faster frequencies are averaged out and the dynamical system is effectively reduced to one degree of freedom, which is integrable. Thus, the solution of the periodically driven system yields the quasi-eigen energy and the quasi-energy eigen states.

\section{QUASI-ENERGY AND QUASI-ENERGY EIGEN STATES}

In order to calculate the quasi- energy eigen states and the quasi-energy of the driven system, we $[22,23]$ make the ansatz that the solution of the Schrödinger equation corresponding to the Hamiltonian (1) is written as,

$$
|\psi(t)\rangle=\sum_{n} C_{n}(t)|n\rangle \exp \left\{-i\left[E_{r}+(n-r) \frac{\hbar}{N}\right] \frac{t}{\hbar}\right\},
$$

where, $\hbar$ is the scaled Planck's constant, and $E_{r}$ is the average energy of the wave-packet in the $N$ th resonance.

We substitute Eq. (2) in the time dependent Schrödinger equation. By following the method of secular perturbation theory, we [23] get the transformed Schrödinger equation as, 
$i \hbar \frac{\partial g}{\partial t}=\left[-\frac{\hbar^{2} N^{2} \zeta}{2} \frac{\partial^{2}}{\partial \theta^{2}}-i N \hbar\left(\omega-\frac{1}{N}\right) \frac{\partial}{\partial \theta}+H_{0}+\lambda V \sin (\theta)\right] \stackrel{\text { IV. THE QUANTUM RECURRENCE TIMES }}{g}$

The scaled parameters, $\zeta=E_{r}^{\prime \prime} / \hbar^{2}$, and, $\omega=E_{r}^{\prime} / \hbar$, physically express the nonlinearity in the system and its frequency, respectively. Here, $E_{r}^{\prime \prime}$, defines the second derivative and $E_{r}^{\prime}$ defines the first derivative of the unperturbed energy, calculated at the mean quantum number, $n=r$.

We apply the method of factorization, such that,

$$
g(\theta, t)=\tilde{g}(\theta) e^{-i \mathcal{E} t / \hbar} e^{-i(\omega-1 / N) \theta / N \zeta \hbar}
$$

and change the variable, $\theta$, as $\theta=2 z+\pi / 2$. The substitutions reduce the Eq. (3) to the standard Mathieu equation [24], viz.,

$$
\left[\frac{\partial^{2}}{\partial z^{2}}+a-2 q \cos (2 z)\right] \tilde{g}(z)=0 .
$$

Here,

$$
a=\frac{8}{N^{2} \zeta \hbar^{2}}\left(\mathcal{E}-\bar{H}_{0}+\frac{(\omega-1 / N)^{2}}{2 \zeta}\right)
$$

where, $\mathcal{E}$ is the quasi-energy of the system. Moreover, $q=4 \lambda V / N^{2} \zeta \hbar^{2}$, and $\tilde{g}(z)$ is a $\pi$-periodic function.

The function $\tilde{g}(\theta)$ is related to $C_{n}(t)$ as,

$$
\begin{aligned}
C_{m} & =\frac{1}{2 \pi} \int_{0}^{2 \pi} g(\varphi) e^{-i(m-r) \varphi} d \varphi \\
& =\frac{1}{2 N \pi} \int_{0}^{2 N \pi} g(\theta) e^{-i(m-r) \theta / N} d \theta .
\end{aligned}
$$

Hence, the Floquet quasi-energy eigen functions are

$$
\left|\psi_{k}(t)\right\rangle=e^{-i \mathcal{E}_{k} t / \hbar}\left|u_{k}(t)\right\rangle,
$$

where, quasi energy eigen values $\mathcal{E}_{k}$, and $\left|u_{k}(t)\right\rangle$ are defined as

$$
\begin{array}{r}
\mathcal{E}_{k} \equiv \frac{\hbar^{2} N^{2} \zeta}{8} a_{\nu(k)}-\frac{(\omega-1 / N)^{2}}{2 \zeta}+\bar{H}_{0}, \\
\left|u_{k}(t)\right\rangle \equiv \frac{1}{2 \pi} \sum_{n} e^{i n t / N} \int_{0}^{2 \pi} d \varphi e^{i \nu N \varphi / 2} e^{-i(n-r) \varphi / 2} P_{\nu(k)}|n\rangle .
\end{array}
$$

An initial excitation produced at the action, $I=I_{0}$, observes various time scales at which it reappears completely or partially during its evolution in the dynamical system. In order to find these time scales at which revivals occur in the quantum mechanical modulated system, we employ the eigen energy $\mathcal{E}_{k}$ of the system [20,26].

These time scales, $T_{\lambda}^{(j)}$, are inversely proportional to the frequencies, $\omega^{(j)}$, where $j$ is an integer. The frequency, $\omega^{(j)}$, is defined as

$$
\omega^{(j)}=(j !)^{-1} \hbar^{(j-1)} \frac{\partial^{(j)} \mathcal{E}_{k}}{\partial I^{(j)}}=(j ! \hbar)^{-1} \frac{\partial^{(j)} \mathcal{E}_{k}}{\partial n^{(j)}},
$$

calculated at $I=I_{0}=n_{0} \hbar$. The index $j$ describes the order of differentiation of the quasi energy, $\mathcal{E}_{k}$. With the increasing values of $j$, we have smaller frequencies which indicate longer times for the higher-order revivals.

The time scale, $T_{\lambda}^{(1)}=T_{\lambda}^{(c l)}$, defines classical period of the driven system and is inversely proportional to $\omega^{(1)}$. In the absence of external periodic modulating force, the frequency $\omega^{(1)}$ reduces to $\omega$.

The time scale, $T_{\lambda}^{(2)}=T_{\lambda}^{(Q)}$, defines quantum mechanical revival time in the modulated systems. It has inverse proportionality with $\omega^{(2)}$. Here, we have $\omega^{(2)}=(2 !)^{-1} \hbar \partial^{2} \mathcal{E}_{k} /\left.\partial I^{2}\right|_{I=I_{0}}$. Partial reappearance of the initially excited wave packet occurs, at the fractions of the revival time. Therefore, it is appropriately named as fractional revival phenomenon.

On substituting the value for the quasi energy, $\mathcal{E}_{k}$, from Eq. (9) in $\omega^{(1)}$, we obtain the classical period as,

$$
T_{\lambda}^{(c l)}=\left[1-M_{o}^{(c l)}\right] T_{0}^{(c l)} \Delta .
$$

By making the same substitution in, $\omega^{(2)}$, we find the quantum revival time for the driven system as,

$$
T_{\lambda}^{(Q)}=\left[1-M_{o}^{(Q)}\right] T_{0}^{(Q)}
$$

Here, the time scales, $T_{0}^{(c l)}(\equiv 2 \pi / \omega)$, and $T_{0}^{(Q)}(\equiv$ $\left.2 \pi\left(\frac{\hbar}{2 !} \zeta\right)^{-1}\right)$, define the classical period and the quantum revival time in the absence of external modulation. Moreover, $\Delta=\left(1-\omega_{N} / \omega\right)^{-1}$ where $\omega_{N}=1 / N$.

The time modification factor $M_{o}^{(c l)}$ and $M_{o}^{(Q)}$ are given as,

$$
M_{o}^{(c l)}=-\frac{1}{2}\left(\frac{\lambda V \zeta \Delta^{2}}{\omega^{2}}\right)^{2} \frac{1}{\left(1-\mu^{2}\right)^{2}}
$$

and 


$$
M_{o}^{(Q)}=\frac{1}{2}\left(\frac{\lambda V \zeta \Delta^{2}}{\omega^{2}}\right)^{2} \frac{3+\mu^{2}}{\left(1-\mu^{2}\right)^{3}}
$$

where,

$$
\mu=\frac{N^{2} \hbar \zeta \Delta}{2 \omega} .
$$

Equations (13) and (14) express the classical period and the quantum revival time in a one degree of freedom system in the presence of an external modulation. These time scales are function of the modulation strength $\lambda$, the frequency, $\omega$, and the nonlinearity, $\zeta$, associated with the unmodulated system.

As the modulation term vanishes, that is $\lambda=0$, the modification terms $M_{o}^{(c l)}$ and $M_{o}^{(Q)}$ disappear. Thus, from Eqs. (13) and (14), it is obvious that the classical period and the quantum revival time in the presence and in the absence of external modulation are equal, that is, $T_{\lambda}^{(c l)}=T_{0}^{(c l)}$ and $T_{\lambda}^{(Q)}=T_{0}^{(Q)}$. As there exist no resonances for $\lambda=0$, we find $\Delta=1$.

\section{CLASSICAL PERIOD AND QUANTUM REVIVAL TIME: INTERDEPENDENCE}

The nonlinearity present in the energy spectrum of the undriven system, contributes to the classical period and the quantum revival time in the presence and in the absence of an external modulating force [26].

\section{A. Vanishing nonlinearity}

In the absence of nonlinearity in the energy spectrum, i.e for $\zeta=0$, the time modification factor for the classical period $M_{o}^{(c l)}$ and for the quantum revival time $M_{o}^{(Q)}$ vanish, which is evident from Eqs. (15) and (16). Thus, a periodically driven linear system displays the quantum revivals after infinite time, i.e $T_{\lambda}^{(Q)}=T_{0}^{(Q)}=\infty$.

Hence, in the modulated linear system only classical period exists. The system displays revivals after the classical period, that is $T_{\lambda}^{(c l)}=T_{0}^{(c l)} \Delta=2 \pi \Delta / \omega$.

\section{B. Weak nonlinearity}

For weakly nonlinear energy spectrum, the classical period, $T_{\lambda}^{(c l)}$, and the quantum revival time, $T_{\lambda}^{(Q)}$, in the presence of modulation are related with, $T_{0}^{(c l)}$, and, $T_{0}^{(Q)}$, of the unmodulated system as,

$$
3 T_{\lambda}^{(c l)} T_{0}^{(Q)}+\Delta T_{0}^{(c l)} T_{\lambda}^{(Q)}=4 \Delta T_{0}^{(Q)} T_{0}^{(c l)} .
$$

Since the quantum revival time, $T_{\lambda}^{(Q)}$, and, $T_{0}^{(Q)}$, depend inversely on nonlinearity in the unmodulated system, they are much larger than the classical period, $T_{\lambda}^{(c l)}$, and $T_{0}^{(c l)}$. Thus, the time modification factors $M_{o}^{(c l)}$ and $M_{o}^{(Q)}$ are related as,

$$
M_{o}^{(Q)}=-3 M_{o}^{(c l)}=-3 \alpha,
$$

where

$$
\alpha=\frac{1}{2}\left(\frac{\lambda V \zeta}{\omega^{2}}\right)^{2}
$$

The modification factor $\alpha$ is directly proportional to the square of the nonlinearity, $\zeta^{2}$ in the system.

In the asymptotic limit, i.e. for $\zeta$ approaching zero, the quantum revival time in the modulated and unmodulated system are equal and infinite, that is $T_{\lambda}^{(Q)}=T_{0}^{(Q)}=\infty$. Furthermore, the classical period in the modulated and unmodulated cases are related as, $T_{\lambda}^{(c l)}=T_{0}^{(c l)} \Delta$, as mentioned above.

The quantum revival time $T_{\lambda}^{(Q)}$ reduces by $3 \alpha T_{0}^{(Q)}$, whereas, $T_{\lambda}^{(c l)}$ increases by $\alpha T_{0}^{(c l)}$.

\section{Strong nonlinearity}

For strongly nonlinear energy spectrum, the classical period, $T_{\lambda}^{(c l)}$, and the quantum revival time, $T_{\lambda}^{(Q)}$, in the presence of the coupling are related with the, $T_{0}^{(c l)}$, and, $T_{0}^{(Q)}$, of the uncoupled system as,

$$
T_{\lambda}^{(c l)} T_{0}^{(Q)}-\Delta T_{0}^{(c l)} T_{\lambda}^{(Q)}=0 .
$$

We have the time modification factors related as

$$
M_{o}^{(Q)}=M_{o}^{(c l)}=-\beta,
$$

where

$$
\beta=\frac{1}{2}\left(\frac{4 \lambda V}{N^{2} \zeta \hbar^{2}}\right)^{2}
$$

Hence, for a highly nonlinear case $\beta$ approaches to zero. Thus, the time modification factors, given in Eqs. (15) and (16), vanish both in the classical domain and in the quantum domain. As a result, the Eqs. (13) and (14) reduces to, $T_{\lambda}^{(c l)}=T_{0}^{(c l)} \Delta$, and $T_{\lambda}^{(Q)}=T_{0}^{(Q)}$, which proves the equality given in Eq. (21).

The quantity $\beta$ which determines the modification both in classical period and in quantum revival time is inversely depending on fourth power of Planck's constant $\hbar$, hence for highly quantum mechanical cases we find that the revival times remain unchanged. 


\section{QUANTUM RECURRENCES AND POWER LAW POTENTIALS}

We may express a large number of potentials as power law potentials [25], such that $V_{1}(z)=V_{0}\left|\frac{z}{a}\right|^{k}$, where $V_{0}$ and $a$ are arbitrary constants. For the corresponding one degree of freedom system, the energy eigen value is

$E_{n}^{(k)}=\left[\left(n+\frac{1}{2}\right) \frac{\pi \hbar}{2 a \sqrt{2 m}} V_{0}^{1 / k} \frac{\Gamma(1 / k+3 / 2)}{\Gamma(1 / k+1) \Gamma(3 / 2)}\right]^{2 k / k+2}$.

The corresponding classical frequency and nonlinearlity in the absence of any external force is defined as

$$
\omega=\frac{1}{\hbar} \frac{2 k}{k+2}\left(r+\frac{1}{2}\right)^{-1} E_{r}^{(k)},
$$

and

$$
\zeta=\frac{1}{\hbar^{2}} \frac{2 k(k-2)}{(k+2)^{2}}\left(r+\frac{1}{2}\right)^{-2} E_{r}^{(k)} .
$$

Hence the classical period and the quantum recurrence time read as

$$
T_{0}^{(c l)}=\frac{2 \pi \hbar}{E_{r}^{(k)}} \frac{k+2}{2 k}\left(r+\frac{1}{2}\right),
$$

and

$$
T_{0}^{(Q)}=\frac{4 \pi \hbar}{E_{r}^{(k)}} \frac{(k+2)^{2}}{2 k(k-2)}\left(r+\frac{1}{2}\right)^{2}
$$

respectively.

We substitute the values of $T_{0}^{(c l)}$ and $T_{0}^{(Q)}$ from Eqs. (27) and (28) in the Eqs. (18) and (21). Thus for a weak nonlinearity in the power law potentials, the interdependence between the classical period and the quantum recurrence time in the presence of external periodic modulation becomes

$$
T_{\lambda}^{(Q)}=-3 C_{k} T_{\lambda}^{(c l)}+4 T_{0}^{(Q)},
$$

and, for a strong nonlinearity it reads as,

$$
T_{\lambda}^{(Q)}=C_{k} T_{\lambda}^{(c l)},
$$

respectively. Here, we define $C_{k}$ as

$$
C_{k}=\frac{2}{\Delta}\left(\frac{k+2}{k-2}\right)\left(r+\frac{1}{2}\right) .
$$

\section{ACKNOWLEDGEMENT}

I submit my thanks to Higher Education Commission, Pakistan, research grant for the research work.

[1] J. H. Eberly, N. B. Narozhny, and J. J. SanchezMondragon, Phys. Rev. Lett. 44, 1323 (1980).

[2] N. B. Narozhny, J. J. Sánchez-Mondragón, and J. H. Eberly, Phys. Rev. A 23 (1981) 236.

[3] B. Yurke, and D. Stoler, Phys. Rev. Lett. 57, 13 (1986).

[4] J. Parker and C. R. Stroud, Jr., Phys. Rev. Lett. 56 (1986) 716.

[5] G. Alber, H. Ritsch, and P. Zoller, Phys. Rev. A 34(1986) 1058.

[6] I. Sh. Averbukh and N.F. Perelman, Phys. Lett. A 139 (1989) 449.

[7] G. Alber and P. Zoller, Phys. Rev. A 37 (1988) 377.

[8] P. A. Braun and V. I. Savichev, J. Phys. B 29 (1996) L329.

[9] C. Leichtle, I. Sh. Averbukh, and W.P. Schleich, Phys. Rev. Lett. 77 (1996) 3999.

[10] C. Leichtle, I. Sh. Averbukh, and W.P. Schleich, Phys. Rev. A 54 (1996) 5299.

[11] D. L. Aronstein and C. R. Stroud, Jr., Phys. Rev. A 62 (2000) 022102.

[12] S. Yu. Grebenshchikov, C. Beck, H. Flöthmann, D. H. Mordaunt and R. Schinke, Chem. Phys. Lett. 271, 197 (1997).

[13] I. Fischer, D. M. Villeneuve, M. J. J. Vrakking, and A. Stolow, J. Chem Phys. 102, 5566 (1995).

[14] M. J. J. Vrakking, D. M. Villeneuve, and A. Stolow, Phys. Rev. A 54, R37 (1996).

[15] M. A. Doncheski, and R. W. Robinett, Ann. Phys. NY 308, 578 (2003).

[16] F. Haake, Quantum signatures of Chaos (Springer, Berlin, 2001).

[17] T. Hogg and B. A. Huberman, Phys. Rev. Lett. 48, 711 (1982).

[18] J. K. Breslin, C.A. Holmes, and G.J. Milburn, Phys. Rev. A 56, 3022 (1997).

[19] S. Tomsovic and J. Lefebvre, Phys. Rev. Lett. 79, 3629 (1997).

[20] F. Saif, and M. Fortunato, Phys. Rev. A 65, 013401 (2002).

[21] M. Born, Mechanics of the atom (Ungar, New York, 1960).

[22] G. P. Berman, and G. M. Zaslavsky, Phys. Lett. A 61 (1977) 295.

[23] M. E. Flatté and M. Holthaus, Ann. Phys. NY 245, 113 (1996).

[24] M. Abramowitz and I.A. Stegun, Handbook of Mathematical Functions, (Dover, New York, 1992).

[25] R. W. Robinett, J. Math. Phys. 41 (2000) 1801.

[26] F. Saif, to be published. 